ISBN 978-981-11-3671-9

Proceedings of 2017 the 7th International Workshop on Computer Science and Engineering

(WCSE 2017)

Beijing, 25-27 June, 2017, pp. 1103-1106

doi: $10.18178 /$ wcse.2017.06.192

\title{
Detection Fuse Blown of Transformer Using GSM
}

\author{
Mian Muhammad Kamal ${ }^{1+}$, Ruonan Zhang and Adnan Ashraf \\ Northwestern Polytechnical University, Xi'an, 710072, P.R. China
}

\begin{abstract}
The main propose of this project is to make a system that automatically detect fuse blown of distribution transformer and inform the central station about the cusses of fuse blown using GSM module. The fuse blown of distribution transformer has three major reasons which are overloading, open circuit and short circuit. The fuse is blown due to overloading if the load (current) on the distribution transformer is exceeds then the maximum limit or safety limit. If the transmission line is damage or cutoff then there will be zero current which will cause fuse blown due to open circuit. When the intersection of transmission line is happened that causes the current will goes to infinity, so the fuse of distribution transformer will be blown due to short circuit. This project consist of two parts hardware and software. The main hardware components to design this circuit are current sensor, micro-controller and GSM module. The software part include the coding of micro-controller using C-programming language. So this project can solve the problems of distribution system and increase the efficiency of distribution system maintenance.
\end{abstract}

Key world: PIC16F873A Microcontrollers, GSMModule, Current Sensor

\section{Introduction}

As with the passage of time the technology is getting more and more advance, it is because of Automation. Now the researcher are doing research to make some automatic circuits so that the machines can run automatically and if the machine occurs any error so it can also give some information about error, which will be so helpful for the fault detection. In distribution transformer the fuses protect the system from overloading whenever there is over loading the fuse is blown and the repair team is called for the correction of fault by any of the affected consumer [1].The automation can make life easier and reduce the work for humans. This thinking make us think about the problem that if the fuse of the distribution transformer is blown then it has two main problems, first there is no such way that can automatically inform the central station about the electricity breakdown. Accurate fault location for electric power lines is critical to improve system availability by reducing restoration time [2].The second one is fault detection or error detection, because the repairing team may take a long time to find the reason of fuse blown. To keep these two problems in mind we make project that can detect the fuse blown of the distribution transformer and also give some useful information about fault detection. There are many reasons for lowvoltage, one of such reasons is the failure in transformer phase(s). Most failures in transformers phases are as a result of overloading [3].The mainly three reasons of fuse damage overloading, open circuit and short circuit. The monitoring devices or systems which are presently used for monitoring distribution transformer have some problems and deficiencies. Few of them are Ordinary transformer measurement system, Detection system itself is not reliable, Timely detection data will not be sent to monitoring centers in time, and a monitoring system can only monitor the operation state [4]. We design a circuit and also

\footnotetext{
${ }^{+}$Corresponding author.

E-mail address: Miankamal@mail.nwpu.edu.cn
} 
use a GSM module that if the fuse is blown then the system automatically send a message to the central station about electricity breakdown and the reason of breakdown like overloading, open circuit or short circuit. Once the massage is received by central station then they will send the repairing team and the problem can be easily resolve on time.

\section{Methodology}

This project is divided into two main parts, one is making a hardware circuits and the other one is software or coding of micro-controller.

\subsection{Hardware circuits:}

In hardware part we can use some important hardware components which are current sensor, microcontroller and GSM module. As we discussed before that there are mainly three reasons of fuse damage overloading, open circuit and short circuit. So we further divide our projects in three parts.

1. Fuse blown due to overloading

2. Fuse blown due to open circuit

3. Fuse blown due to short circuit

First of all we make the circuit that can detect the fuse blown of distribution transformer as shown in Fig 1. This circuit is connected to the secondary side of distribution transformer to detect the fuse blown.

\subsubsection{Fuse blown due to overloading:}

When the fuse is blown the current sensor will measure the current and send the measurement (signal) to the micro-controller. The micro-controller will compare the current value with some reference value of current and decide the reason of flue blown. If the measurement is exceed form the safety limit $(0.45 \mathrm{~A}$ to $0.89 \mathrm{~A})$ shown in Fig 2.The micro-controller will send the overloading signal to the GSM module. The GSM module will send the message "fuse is blown due to overloading" to the central station. The GSM module will also send the address (location) of distribution transformer.

\subsubsection{Fuse blown due to open circuit}

As the current sensor is continuously measuring the current. So when the transmission line is damage or cut off due to any reason, the current sensor will sense the zero current or no current shown in Fig3. The current sensor will send the measurement of current to the micro-controller. The micro-controller will compare the current reading with some reference and send the open circuit signal to the GSM module. Then the GSM module will send the message "fuse is blown due to open circuit" to the central station as well as the location of the distribution transformer.

\subsubsection{Fuse blown due to short circuit}

The transmission line has three wires Phase, Neutral and Ground. When the Phase and Neutral wires are intersect with each other directly without any load between them then the fuse of distribution transformer is blown due to short circuit. In this case the current goes to infinity ( 0.9 to infinity) and the measurement of current sensor will reach to its maximum range shown in Fig4.The current sensor will send the signal of current measurement to the micro-controller. The micro-controller will decide the cause of fuse blown is short circuit and pass the signal to the GSM module. So the GSM module will send the message "fuse is blown due to short circuit" and location of transformer to the central station.

\subsection{Software or Micro-Controller Coding}

The second main part of this project is the coding of micro-controller. The C-programming language is used to write a code. Micro-controller needs some instruction to perform specific task. So for the purpose of making decision by micro-controller automatically, we make a program that give some instructions and reference values 
to the micro-controller. The micro-controller take the measurement form current sensor, compare with already stored reference values and take a decision about fuse blown due to overloading, open circuit or short circuit.

\section{Main Component}

1. Power supply

2. IC LUN2803

3. GSM Module

4. Current Sensor

5. Voltage Regulator (7805)

6. DC Adaptor

7. LCD

8. PIC16F873A Micro-Controller

\section{Block Diagram}
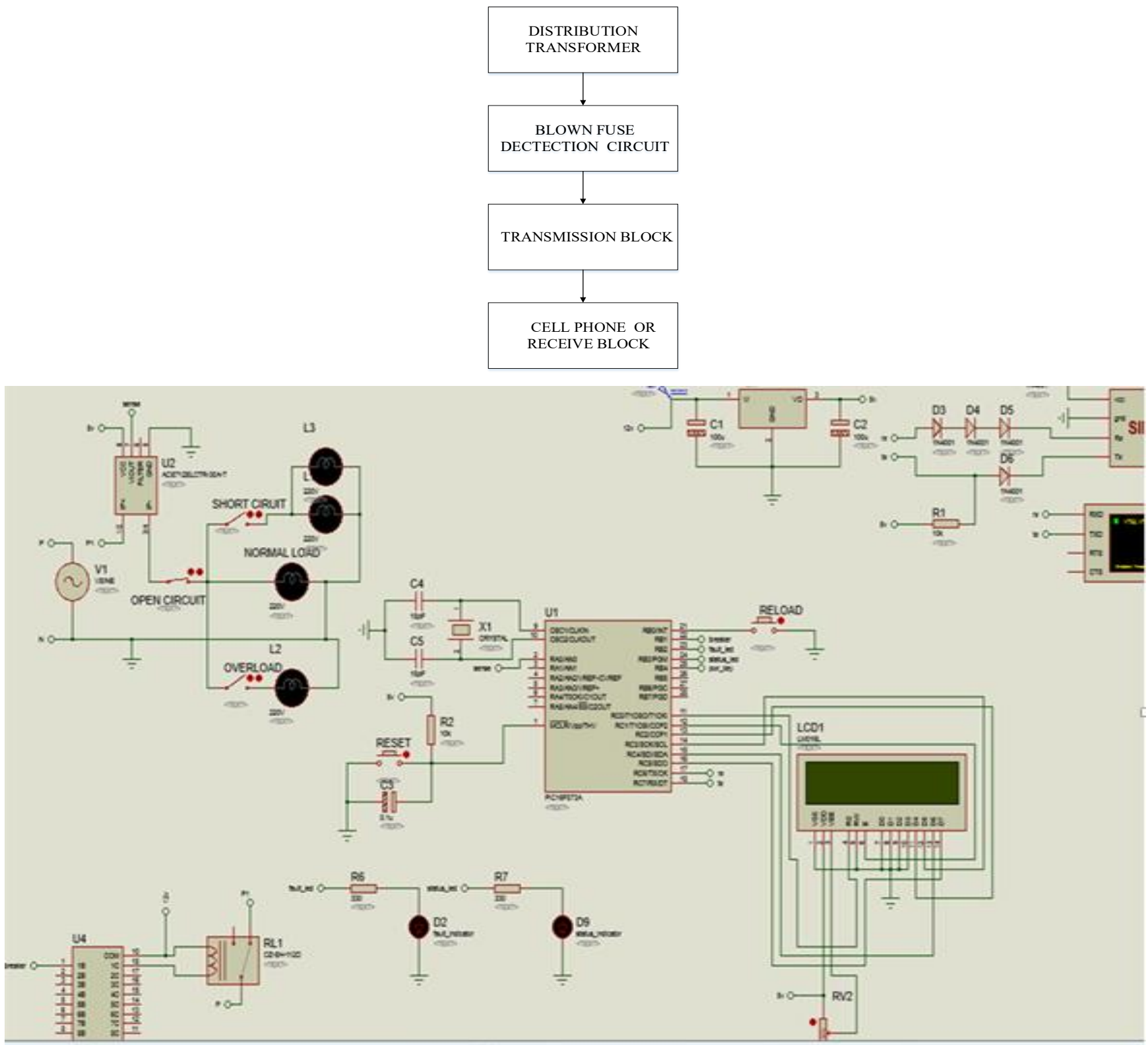

Fig 1: Schematic Circuit 


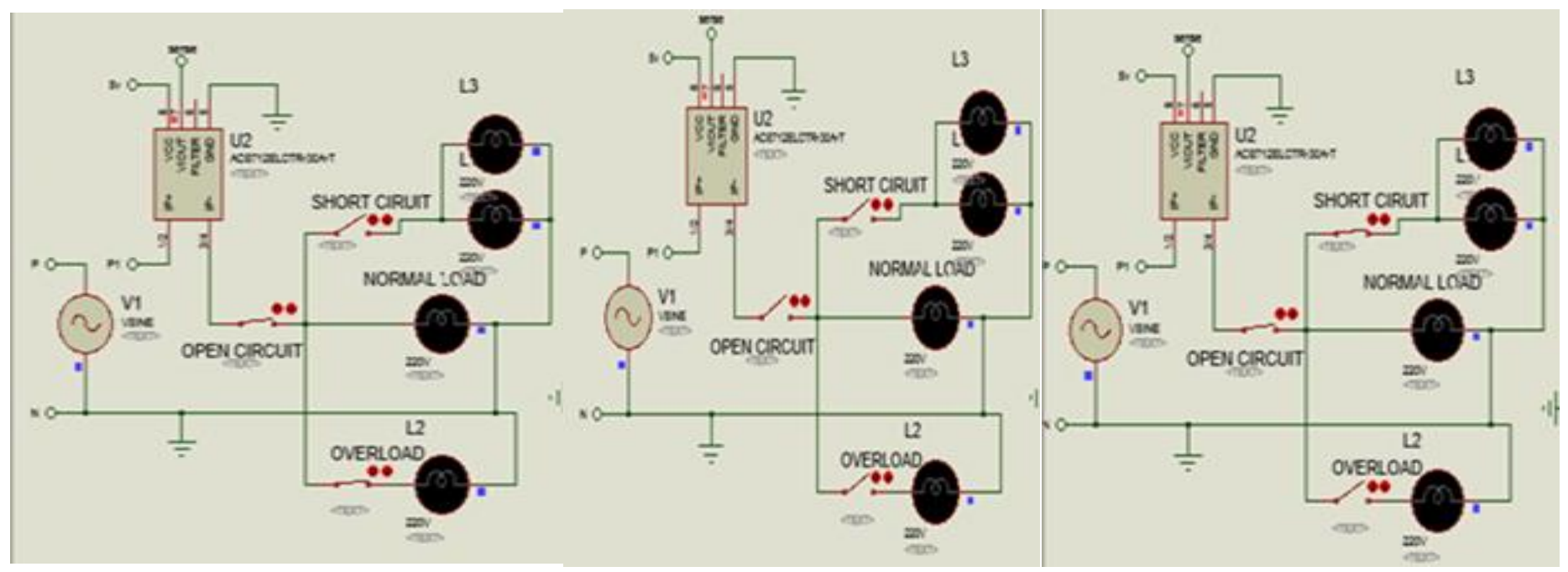

Fig2: Overload Circuit

Fig 3: Open Circuit

Fig4: Short Circuit

\section{Result}

Before when there is an electricity breakdown the central station was informed by the customer that are been affected and also they do not have any useful information about fault detection. Due to which the reappearing team need more time to detect and correct the fault. Our system not even can automatically inform the central station about the electricity breakdown but also inform the central station by using GSM module about the reason of breakdown like overloading, open circuit or short circuit. The previous system have no facility to inform automatically about the reasons of electricity breakdown.

\section{Conclusion}

This paper discusses the automatically detection of fuse blown of distribution transformer and inform the central station about the cusses of fuse blown using GSM module. This technique is reliable and very useful in real life to improve the maintenance efficiency of distribution system. This system can also be used in industry to find the fault of electricity breakdown automatically. In future we can also use power line communication to transfer the information about fault detection to the central station.

\section{References:}

[1] Mian Muhammad Kamal "GSM Based Link Monitoring of Distribution Transformer ", International Journal of Recent Research Aspects ISSN: 2349- 7688, vol. 2, Issue 4,pp.56-59, December 2015.

[2] CHEN Ping, WANG Kuixin, "Fault location technology for high-voltage overhead lines combined with underground power cables based on travelling wave principle". International Conference on Advanced Power System Automation and Protection, Beijing, China, pp.748-751,Oct16-20,2011.

[3] J. O. Egwaile and N. Bello, "Design and Implementation of GSM Based Transformer Phase Monitoring System", Nigerian Journal of Technology (NIJOTECH) vol. 33. No. 3,pp. 331 - 336, July 2014.

[4] Nikhil P Shetty, SingriAnirudha Prasad, YashSachdeva, Ashiq Ali B Y, TruptiTagare, "Transformer Monitoring System Using GSM Module" international journal of innovative research in electrical, electronics, instrumentation and control engineering vol. 4, issue 5,pp.221-223, May 2016, 